\title{
(Nano)particle \\ classification/identification using single particle ICP-Time of Flight-MS acquisition coupled to cluster analysis
}

\author{
MICKAEL THARAUD ${ }^{1}$, LUKAS SCHLATT ${ }^{2}$, PHIL SHAW $^{2}$ \\ AND DR. MARC F. BENEDETTI ${ }^{3}$ \\ ${ }^{1}$ IPGP-CNRS UMR 7154 \\ ${ }^{2} \mathrm{Nu}$ Instruments \\ ${ }^{3}$ IPGP-CNRS UMR 7154 -Université de Paris \\ Presenting Author: tharaud@ipgp.fr
}

spICP-MS where a single isotope signal can be acquired continuously at dwell times between $10-100 \mu$ s is a particularly helpful technique when investigating the number and size of of individual elemental nanoparticles. The technique is improved upon when using an ICP-Time of Flight (ToF)-MS as the multielement compositions of each particle can be determined. The ICP-ToF-MS is further optimized for the single particle technique when able to continuously collect the ion beam at a few tens of $\mu$ s per spectra with no interruption as it allows

i/ the complete evaluation of each NP signal

ii/ collecting all NP signals without loss

iii/ determining the (multi)-elemental composition of NPs individually

This makes the information on the NP population in the sample more thorough and reliable compare to information provided using a conventional single isotope ICP-MS method. Indeed, single particle composition is given simultaneously, and part of the interest of using a multi-elemental spICP-MS analysis is its potential for the identification/classification of natural or synthetic compounds for the determination of the risk assessment and of the possible health hazard. In this study, the signal of mono-, bi- and tri-metallic synthetic nanoparticles either alone or mixed will be analysed using a novel ICP-tof-MS with the desired performance characteristics (Nu Instruments Vitesse) and multiple ways to examine the data produced will be explored.

In addition, natural particles including aluminium oxide, montmorillonite and kaolinite which are particles now dispersed in the environment due to the increase of anthropogenic activities (ie. soil erosion in the critical zone), will be investigated. Finally, the applicability to breathable inorganic particles and/or elongate mineral particles (EMP) will be demonstrated. 